\title{
On simplicial maps of the complexes of curves of nonorientable surfaces
}

\author{
ELMAS IRMAK
}

\begin{abstract}
Let $N$ be a compact, connected, nonorientable surface of genus $g$ with $n$ boundary components and $\mathcal{C}(N)$ be the complex of curves of $N$. Suppose that $g+n \leq 3$ or $g+n \geq 5$. If $\lambda: \mathcal{C}(N) \rightarrow \mathcal{C}(N)$ is an injective simplicial map, then $\lambda$ is induced by a homeomorphism of $N$. If $(g, n) \neq(1,2)$ and $\lambda: \mathcal{C}(N) \rightarrow \mathcal{C}(N)$ is a simplicial map that satisfies the connectivity property, then $\lambda$ is induced by a homeomorphism of $N$.
\end{abstract}

57M99; 20F38

\section{Introduction}

This paper combines the proofs and the results given by the author in $[13 ; 8]$ about simplicial maps of complexes of curves on nonorientable surfaces. The complex of curves of a compact, connected, orientable surface was defined as an abstract simplicial complex by Harvey in [7] as follows: The vertex set consists of isotopy classes of nontrivial simple closed curves, where nontrivial means it does not bound a disk and it is not isotopic to a boundary component of the surface. Vertices form a simplex if they can be represented by pairwise disjoint simple closed curves on the surface. For a compact, connected, nonorientable surface $N$, the complex of curves $\mathcal{C}(N)$ is defined similarly where a simple closed curve is called nontrivial if it does not bound a disk or a Möbius band, and it is not isotopic to a boundary component of the surface. Simplicial maps such as automorphisms, superinjective simplicial maps and injective simplicial maps of the complexes of curves on surfaces are studied to get information about the mapping class groups of these surfaces. The mapping class group is defined as the group of isotopy classes of all self-homeomorphisms of the surface.

On nonorientable surfaces the author proved that each superinjective simplicial map of the complex of curves is induced by a homeomorphism in [14]. The referee of that paper suggested that a stronger result should hold: That if a simplicial map of the curve complex satisfies the connectivity property, ie "two vertices are connected by an edge if and only if their images are connected by an edge", then it should be induced by a homeomorphism. The author proved the referee's suggestion in [13]. This is our 
Theorem 1.2 given below. This result implies that each superinjective simplicial map of the complex of curves is induced by a homeomorphism. It also implies that each automorphism of the complex of curves is induced by a homeomorphism. After that the author proved that each injective simplicial map of the complex of curves is also induced by a homeomorphism in [8]. This is our Theorem 1.1 given below. This result improved all of the above results about simplicial maps of complexes of curves on nonorientable surfaces.

The main results of this paper are the following:

Theorem 1.1 Let $N$ be a compact, connected, nonorientable surface of genus $g$ with $n$ boundary components. Suppose that $g+n \leq 3$ or $g+n \geq 5$. If $\lambda: \mathcal{C}(N) \rightarrow \mathcal{C}(N)$ is an injective simplicial map, then $\lambda$ is induced by a homeomorphism $h: N \rightarrow N$ (ie, $\lambda([a])=[h(a)]$ for every vertex $[a]$ in $\mathcal{C}(N))$.

Theorem 1.2 Let $N$ be a compact, connected, nonorientable surface of genus $g$ with $n$ boundary components. Suppose that either

$$
(g, n) \in\{(1,0),(1,1),(2,0),(2,1),(3,0)\} \quad \text { or } \quad g+n \geq 5 .
$$

If $\lambda: \mathcal{C}(N) \rightarrow \mathcal{C}(N)$ is a simplicial map that satisfies the connectivity property, then $\lambda$ is induced by a homeomorphism $h: N \rightarrow N$.

Here are some known results on compact, connected, orientable surfaces: Ivanov proved that each automorphism of the complex of curves is induced by a homeomorphism [18]. By using this result he classified isomorphisms between any two finite index subgroups of the extended mapping class groups [18]. Korkmaz proved Ivanov's results for lower genus cases [26], and Luo gave a proof for all cases [27]. Ivanov and McCarthy classified injective homomorphisms between mapping class groups [19]. The mapping class group is isomorphic to the automorphism group of several complexes on orientable surfaces. These isomorphisms are given for complexes such as the complex of pants decompositions (proven by Margalit [28]), the complex of nonseparating curves (proven by the author [10]), the complex of separating curves (proven by Brendle and Margalit [5], and McCarthy and Vautaw [29]), the complex of Torelli geometry (proven by Farb and Ivanov [6]), the Hatcher-Thurston complex (proven by Irmak and Korkmaz [16]), and the complex of arcs (proven by Irmak and McCarthy [17]). Farb and Ivanov obtained applications showing that the automorphism group of the Torelli subgroup is isomorphic to the mapping class group [6]. This result was extended by McCarthy and Vautaw to genus at least 3 [29].

Superinjective simplicial maps were defined by the author in [9] on compact, connected orientable surfaces as simplicial maps of the complexes of curves that preserve geometric 
intersection zero and nonzero properties of the vertices. The author proved that each superinjective simplicial map is induced by a homeomorphism of the surface. As an application, she gave a classification of injective homomorphisms from finite index subgroups of the extended mapping class group to the extended mapping class group for genus at least two $[9 ; 11 ; 10]$. Behrstock and Margalit, and Bell and Margalit, proved the author's results for small genus cases in [3] and [4], respectively. Brendle and Margalit proved that if $K$ is the subgroup of mapping class group generated by Dehn twists about separating curves, then any injection from a finite index subgroup of $K$ to the Torelli group is induced by a homeomorphism [5]. They obtained this as an application of another result, after proving that each superinjective simplicial map of the separating curve complex is induced by a homeomorphism. Shackleton proved that each injective simplicial map is also induced by a homeomorphism. He obtained strong local co-Hopfian results as applications [31].

Kida proved several results about superinjective simplicial maps on orientable surfaces in $[20 ; 21 ; 22]$, and as applications he proved that for all but finitely many compact orientable surfaces the abstract commensurators of the Torelli group and the Johnson kernel for such surfaces are naturally isomorphic to the extended mapping class group, any injective homomorphism from a finite index subgroup of the Johnson kernel into the Torelli group for such a surface is induced by an element of the extended mapping class group, and any finite index subgroup of the Johnson kernel is co-Hopfian. Irmak, Ivanov and McCarthy proved that each automorphism of a surface braid group is induced by a homeomorphism of the underlying surface, provided that this surface is a closed, connected, orientable surface of genus at least 2, and the number of strings is at least three in [15]. Kida and Yamagata also proved several results about superinjective simplicial maps in $[23 ; 24 ; 25]$, and as applications they gave a description of any injective homomorphism from a finite index subgroup of the pure braid group with $n$ strands on a closed orientable surface of genus $g$ into the pure braid group. They proved that the abstract commensurator of the braid group with $n$ strands on a closed orientable surface of genus $g$ is naturally isomorphic to the extended mapping class group of a compact orientable surface of genus $g$ with $n$ boundary components. They also proved that for a connected, compact and orientable surface of genus two with one boundary component, any finite index subgroup of the Torelli group is co-Hopfian.

Mapping class groups and abstract simplicial complexes on nonorientable surfaces are not studied as much as the orientable case. Here are some known results for nonorientable surfaces: Atalan proved that the automorphism group of the curve complex is isomorphic to the mapping class group for most odd genus cases [1]. The author proved that each injective simplicial map of the complex of arcs is induced by a homeomorphism, and the automorphism group of the complex of arcs is isomorphic 
to the mapping class group in most cases [12]. Atalan and Korkmaz proved that the automorphism group of the curve complex is isomorphic to the mapping class group for most cases [2]. They also proved that two curve complexes are isomorphic if and only if the two surfaces they are defined on are homeomorphic. The author proved that each superinjective simplicial map of the complex of curves is induced by a homeomorphism in most cases [14].

The results given in this paper improve all of the above results about simplicial maps of the complexes of curves on nonorientable surfaces. By using these results the author hopes to give a classification of the injective homomorphisms of the mapping class groups on nonorientable surfaces. We note that for the results given in this paper the case when $g+n=4$ is open.

\section{Injective simplicial maps}

In this section we will assume that $N$ is a compact, connected, nonorientable surface of genus $g$ with $n$ boundary components. We will work on pair of pants decompositions on $N$. We define them as follows: If $a$ is a simple close curve on $N$, let $N_{a}$ be the cut surface along $a$. A set, $P$, of pairwise disjoint, nonisotopic, nontrivial simple closed curves on $N$ is called a pair of pants decomposition of $N$ if each component of $N_{P}$ is a pair of pants. The set of isotopy classes of elements of $P$ forms the vertices of a maximal simplex of $\mathcal{C}(N)$. Every maximal simplex of $\mathcal{C}(N)$ is obtained by the isotopy classes of elements of some pair of pants decomposition of $N$. All maximal simplices in the complexes of curves have the same dimension on orientable surfaces, but $\mathcal{C}(N)$ has different-dimensional maximal simplices. We show several pants decompositions on a closed surface of genus 7 in Figure 1. These pants decompositions correspond to maximal simplices in $\mathcal{C}(N)$. They have different dimensions. There are cross signs in the figures. This means that we remove the interiors of the disks that have cross signs in them, and then we identify the antipodal points of the resulting boundary components.

The following lemma is given in $[1 ; 2]$ :

Lemma 2.1 Let $N$ be a nonorientable surface of genus $g \geq 2$ with $n$ boundary components. Suppose that $(g, n) \neq(2,0)$. Let $a_{r}=3 r+n-2$ and $b_{r}=4 r+n-2$ if $g=2 r+1$, and let $a_{r}=3 r+n-4$ and $b_{r}=4 r+n-4$ if $g=2 r$. Then there is a maximal simplex of dimension $q$ in $\mathcal{C}(N)$ if and only if $a_{r} \leq q \leq b_{r}$.

Let $\Delta$ be a maximal simplex in $\mathcal{C}(N) . \Delta$ will be called a top-dimensional maximal simplex if $\Delta$ has the highest possible dimension. 


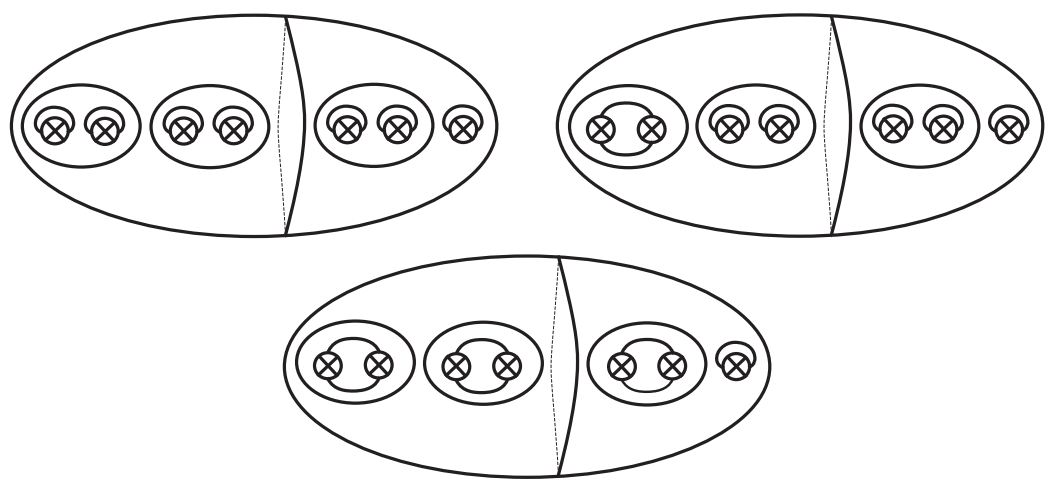

Figure 1: Pants decompositions

Lemma 2.2 Let $g \geq 2$. Suppose that $(g, n)=(3,0)$ or $g+n \geq 4$. Let $P$ be pair of pants decompositions which correspond to a top-dimensional maximal simplex in $\mathcal{C}(N)$. The curves in $P$ are either separating or $1-$ sided with nonorientable complements. In $P$, the number of 1 -sided curves whose complements are nonorientable is $g$, and the number of separating curves is $2 r+n-2$ if $g=2 r+1$, and $2 r+n-3$ if $g=2 r$.

Proof Let $P$ be a pair of pants decomposition that corresponds to a top-dimensional maximal simplex, $\Delta$, in $\mathcal{C}(N)$. Let $a \in P$. If $a$ is a 1 -sided simple closed curve whose complement is orientable, then the genus of $N$ is odd, say $g=2 r+1$ for some $r \in \mathbb{Z}$. We see that $r \geq 1$ as $g \geq 2$. The complement of $a$ is an orientable surface of genus $r$ with $n+1$ boundary components. On an orientable surface of genus $g_{0}$ with $n_{0}$ boundary components, all maximal simplices have dimension $3 g_{0}+n_{0}-4$. This implies that $[a]$ can be a vertex of at most a $(3 r+n-2)$-dimensional simplex in $\mathcal{C}(N)$. Since the dimension of $\Delta$ is $4 r+n-2$ by Lemma 2.1 and $r \geq 1$, we get a contradiction. So, $a$ is not a 1 -sided simple closed curve whose complement is orientable.

Suppose that $a$ is a 2-sided nonseparating simple closed curve. Case (i): Suppose $g=2 r$ for some $r \in \mathbb{Z}$. Then $r \geq 1$ as $g \geq 2$. If the complement of $a$ is nonorientable, then it has genus $2 r-2$ and $n+2$ boundary components. Then by Lemma 2.1, there is at most a $(4 r+n-5)$-dimensional simplex containing $[a]$ as a vertex in $\mathcal{C}(N)$. Since the dimension of $\Delta$ is $4 r+n-4$ by Lemma 2.1, we get a contradiction. If the complement of $a$ is orientable, then the complement is an orientable surface of genus $r-1$ with $n+2$ boundary components. In this case there is at most a $(3 r+n-4)$-dimensional simplex containing $[a]$ in $\mathcal{C}(N)$. Since the dimension of $\Delta$ is $4 r+n-4$ by Lemma 2.1 and $r \geq 1$, we get a contradiction. Case (ii): Suppose $g=2 r+1$ for some $r \in \mathbb{Z}$. In this case complement of $a$ is a nonorientable surface 
of genus $2 r-1$ with $n+2$ boundary components. By Lemma 2.1, there is at most a $(4 r+n-3)$-dimensional simplex containing $[a]$ in $\mathcal{C}(N)$. Since the dimension of $\Delta$ is $4 r+n-2$ by Lemma 2.1, we get a contradiction. So, $a$ is not a 2 -sided nonseparating simple closed curve on $N$.

Hence, the curves in $P$ are either separating or 1-sided with nonorientable complements. Gluing pairs of pants along curves that come from cutting along separating curves on the surface does not give a nonorientable surface. So, in $P$ the number of 1 -sided curves with nonorientable complements is $g$. Hence, by using Lemma 2.1, we see that in $P$ the number of separating curves is $2 r+n-2$ if $g=2 r+1$, and $2 r+n-3$ if $g=2 r$.

Let $a$ and $b$ be two nonisotopic nontrivial simple closed curves such that they have nonzero geometric intersection, $i([a],[b]) \neq 0$. We will say that $a$ and $b$ have small intersection if there exists a pair of pants decomposition $P$ on $N$ that corresponds to a top-dimensional maximal simplex in $\mathcal{C}(N)$ such that $a \in P$ and $(P \backslash\{a\}) \cup\{b\}$ also corresponds to a top-dimensional maximal simplex in $\mathcal{C}(N)$.

Lemma 2.3 Suppose that $g+n \geq 4$. Let $\lambda: \mathcal{C}(N) \rightarrow \mathcal{C}(N)$ be an injective simplicial map. If $a$ and $b$ have small intersection, then $i(\lambda([a]), \lambda([b])) \neq 0$.

Proof Suppose $a$ and $b$ have small intersection. We complete $a$ to a pair of pants decomposition $P$ on $N$ that corresponds to a top-dimensional maximal simplex in $\mathcal{C}(N)$ such that $(P \backslash\{a\}) \cup\{b\}$ also corresponds to a top-dimensional maximal simplex in $\mathcal{C}(N)$. Let $P^{\prime}$ be a set of pairwise disjoint curves representing $\lambda([P])$. Since $\lambda$ is injective, $P^{\prime}$ corresponds to a top-dimensional maximal simplex. Since $b$ does not intersect any of the curves in $P \backslash\{a\}, i(\lambda([b], \lambda([x]))=0$ for any $x \in P \backslash\{a\}$. Since $\lambda$ is injective, $\lambda([b])$ is not equal to $\lambda([y])$ for any $y \in P$. Then, since $P^{\prime}$ corresponds to a top-dimensional maximal simplex, we see that $i(\lambda([a]), \lambda([b])) \neq 0$.

Let $a$ and $b$ be two distinct elements in a pair of pants decomposition $P$ on $N$ where $P$ corresponds to a top-dimensional maximal simplex in $\mathcal{C}(N)$. Then $a$ is called adjacent to $b$ with respect to $P$ if there exists a pair of pants in $P$ which has $a$ and $b$ on its boundary. In the following lemmas we will see that adjacency and nonadjacency are preserved with respect to top-dimensional maximal simplices.

Lemma 2.4 Suppose that $g+n \geq 4$. Let $\lambda: \mathcal{C}(N) \rightarrow \mathcal{C}(N)$ be an injective simplicial map. Let $P$ be a pair of pants decomposition on $N$ which corresponds to a topdimensional maximal simplex in $\mathcal{C}(N)$. Let $a, b \in P$ such that $a$ is not adjacent to $b$ with respect to $P$. There exists $a^{\prime} \in \lambda([a])$ and $b^{\prime} \in \lambda([b])$ such that $a^{\prime}$ is not adjacent to $b^{\prime}$ with respect to $P^{\prime}$ where $P^{\prime}$ is a set of pairwise disjoint curves representing $\lambda([P])$ containing $a^{\prime}, b^{\prime}$. 


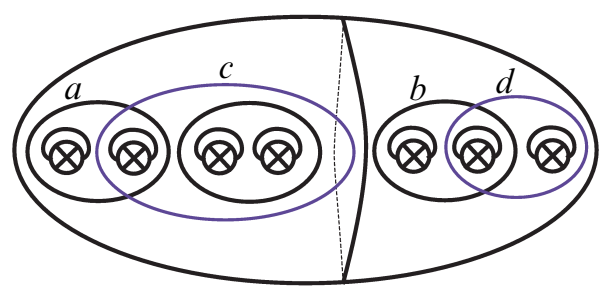

(i)

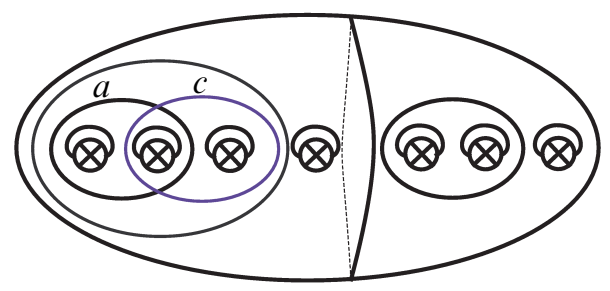

(iii)

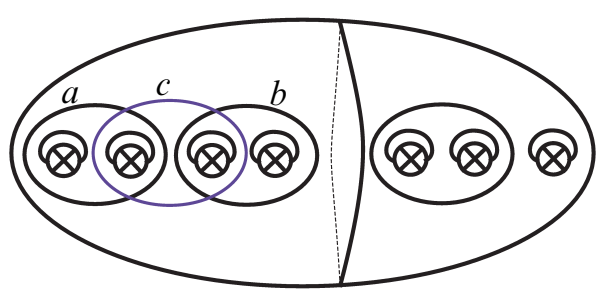

(ii)

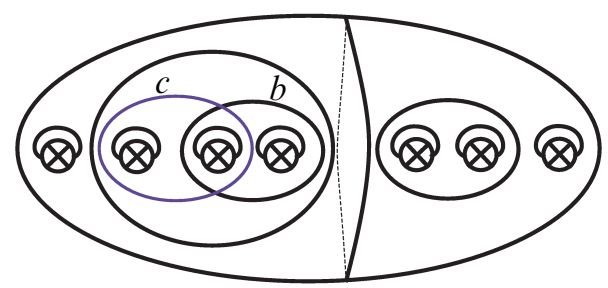

(iv)

Figure 2: Curves on closed surface of genus 7

Proof Let $a$ and $b$ be two curves in $P$. Assume that $a$ is not adjacent to $b$ with respect to $P$. We can choose simple closed curves $c, d$ on $N$ such that $c$ and $a$ have small intersection, $c$ does not intersect any of the curves in $P \backslash\{a\}, d$ and $b$ have small intersection, $d$ does not intersect any of the curves in $P \backslash\{b\}, c$ and $d$ are disjoint, and $a, b, c, d$ are all pairwise nonisotopic. In Figure 2(i), we show how to choose $c, d$ for a special case on a closed surface of genus 7 , where $a, b, P$ are as shown in the figure. Let $a^{\prime} \in \lambda([a])$ and $b^{\prime} \in \lambda([b])$. We choose a set of pairwise disjoint simple closed curves representing $\lambda([P])$ containing $a^{\prime}, b^{\prime}$, call it $P^{\prime}$. By using Lemma 2.3 we see that $i(\lambda([a]), \lambda([c])) \neq 0, i(\lambda([b]), \lambda([d])) \neq 0$. Since $\lambda$ is injective we also know that none of the two elements in $\{\lambda([a]), \lambda([b]), \lambda([c]), \lambda([d])\}$ are equal. Since $c$ does not intersect any of the curves in $P \backslash\{a\}$, we see that $\lambda([c])$ has geometric intersection zero with any element in $\lambda([P]) \backslash\{\lambda([a])\}$. Similarly, $\lambda([d])$ has geometric intersection zero with any element in $\lambda([P]) \backslash\{\lambda([b])\}$. Since $i([c],[d])=0$, we see that $i(\lambda([c]), \lambda([d]))=0$. This is possible only when $a^{\prime}$ is not adjacent to $b^{\prime}$ with respect to $P^{\prime}$.

Lemma 2.5 Suppose that $(g, n)=(1,4)$ or $(2,2)$. Let

$$
\lambda: \mathcal{C}(N) \rightarrow \mathcal{C}(N)
$$

be an injective simplicial map. If $a$ is a 1 -sided simple closed curve on $N$, then there exists $a^{\prime} \in \lambda([a])$ such that $a^{\prime}$ is a 1-sided simple closed curve on $N$. 
Proof Let $a$ be a 1 -sided simple closed curve. If $(g, n)=(1,4)$, we complete $a$ to a pants decomposition $P=\{a, z, t\}$ as shown in Figure 3(i). Let $a^{\prime} \in \lambda([a]), z^{\prime} \in \lambda([z])$, $t^{\prime} \in \lambda([t])$ and $a^{\prime}, z^{\prime}, t^{\prime}$ have minimal intersection. Let $P^{\prime}=\left\{a^{\prime}, z^{\prime}, t^{\prime}\right\} . P^{\prime}$ corresponds a top-dimensional maximal simplex in $\mathcal{C}(N)$. Since $a$ is not adjacent to $t$, and nonadjacency is preserved by Lemma 2.4, we see that $z^{\prime}$ has to be a separating curve, $a^{\prime}$ and $t^{\prime}$ have to be on different sides of $z^{\prime}$. Suppose $a^{\prime}$ is a separating curve. Then $a^{\prime}, z^{\prime}, t^{\prime}$ are as shown in Figure 3(ii). Then, we get a contradiction by using the curves $x, y$ given in part (i). The curves $x, y, a, z, t$ are pairwise nonisotopic and each $x$ and $y$ has small intersection with $t$, and disjoint from $z$. Let $x^{\prime}, y^{\prime}$ be representatives of $\lambda([x])$ and $\lambda([y])$ that have minimal intersection with each of $a^{\prime}, z^{\prime}, t^{\prime}$. Since $\lambda$ is injective, $x^{\prime}, y^{\prime}, a^{\prime}, z^{\prime}, t^{\prime}$ are pairwise nonisotopic. By using Lemma 2.3, we see that $x^{\prime}$ and $y^{\prime}$ should intersect $t^{\prime}$ and should be disjoint from $z^{\prime}$. This gives a contradiction. So, $a^{\prime}$ is not a separating curve. Since $g=1, a^{\prime}$ is a 1 -sided curve.

If $(g, n)=(2,2)$, we complete $a$ to a pants decomposition $P=\{a, y, b\}$ as shown in Figure 3(iii). Let $a^{\prime} \in \lambda([a]), y^{\prime} \in \lambda([y]), b^{\prime} \in \lambda([b])$ and $a^{\prime}, y^{\prime}, b^{\prime}$ have minimal intersection. Let $P^{\prime}=\left\{a^{\prime}, y^{\prime}, b^{\prime}\right\} . P^{\prime}$ corresponds to a top-dimensional maximal simplex. By Lemma 2.2, we know that $a^{\prime}$ is either a separating curve or a 1 -sided curve. Since $a$ is not adjacent to $b$ with respect to $P$, and nonadjacency is preserved by Lemma 2.4 , we see that $y^{\prime}$ has to be a separating curve, and $a^{\prime}$ and $b^{\prime}$ have to be on different sides of $y^{\prime}$. This implies that $a^{\prime}, y^{\prime}, b^{\prime}$ are as shown in Figure 3(iv). Hence, $a^{\prime}$ is a 1 -sided curve.

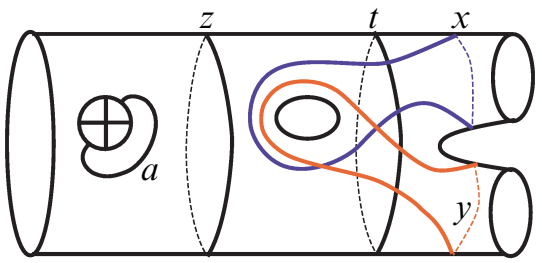

(i)

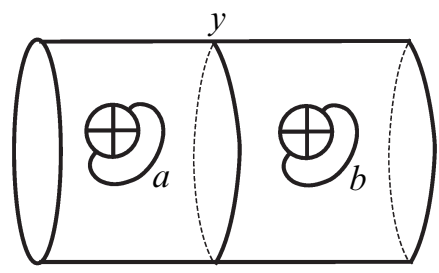

(iii)

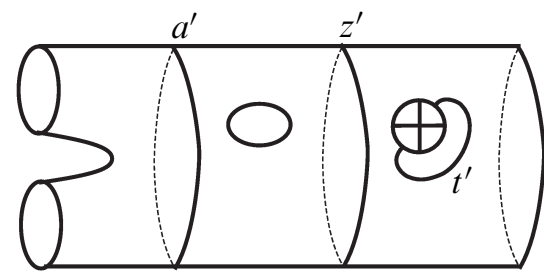

(ii)

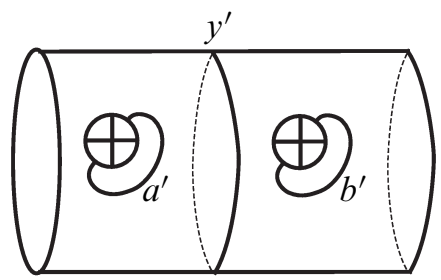

(iv)

Figure 3: Curve configurations I 
Lemma 2.6 Suppose that $g+n \geq 5$. Let $\lambda: \mathcal{C}(N) \rightarrow \mathcal{C}(N)$ be an injective simplicial map. Let $P$ be a pair of pants decomposition on $N$ that corresponds to a top-dimensional maximal simplex in $\mathcal{C}(N)$. Let $a, b \in P$ such that $a$ and $b$ are both $2-$ sided and $a$ is adjacent to $b$ with respect to $P$. There exists $a^{\prime} \in \lambda([a])$ and $b^{\prime} \in \lambda([b])$ such that $a^{\prime}$ is adjacent to $b^{\prime}$ with respect to $P^{\prime}$ where $P^{\prime}$ is a set of pairwise disjoint curves representing $\lambda([P])$ containing $a^{\prime}, b^{\prime}$.

Proof Let $a, b \in P$ such that $a$ and $b$ are both 2-sided and $a$ is adjacent to $b$ with respect to $P$. We can choose a simple closed curve $c$ on $N$ such that $a$ and $c$ have small intersection, $b$ and $c$ have small intersection, and $c$ does not intersect any other curve in $P$. In Figure 2(ii) we show how to choose the curve $c$ for a special case on a closed surface of genus 7, where $a, b, P$ are shown in the figure. In Figure 2(iii) we show why $a$ and $c$ have small intersection. In Figure 2(iv) we show why $b$ and $c$ have small intersection. By using Lemma 2.3, we see that $\lambda([a])$ and $\lambda([c])$ have nonzero geometric intersection, and $\lambda([b])$ and $\lambda([c])$ have nonzero geometric intersection. We also see that $\lambda([c])$ has zero geometric intersection with all the other elements in $\lambda([P])$. This implies that there exists $a^{\prime} \in \lambda([a])$ and $b^{\prime} \in \lambda([b])$ such that $a^{\prime}$ is adjacent to $b^{\prime}$ with respect to $P^{\prime}$ where $P^{\prime}$ is a set of pairwise disjoint curves representing $\lambda([P])$ containing $a^{\prime}, b^{\prime}$.

In the following lemmas we show that adjacency is preserved for other types of simple closed curves.

Lemma 2.7 Let $g \geq 2$. Suppose that $(g, n)=(3,0)$ or $g+n \geq 4$. Let $\lambda: \mathcal{C}(N) \rightarrow$ $\mathcal{C}(N)$ be an injective simplicial map. Let $P$ be a pair of pants decomposition on $N$ that corresponds to a top-dimensional maximal simplex in $\mathcal{C}(N)$. Let $a, b \in P$ such that $a$ and $b$ are both 1 -sided curves and $a$ is adjacent to $b$ with respect to $P$. There exists $a^{\prime} \in \lambda([a])$ and $b^{\prime} \in \lambda([b])$ such that $a^{\prime}$ is adjacent to $b^{\prime}$ with respect to $P^{\prime}$, where $P^{\prime}$ is a set of pairwise disjoint curves representing $\lambda([P])$ containing $a^{\prime}, b^{\prime}$.

Proof Suppose that $(g, n)=(3,0)$ or $g+n \geq 4$. Let $a, b \in P$ such that $a$ and $b$ are both $1-$ sided, and $a$ is adjacent to $b$ with respect to $P$. Let $P^{\prime}$ be a set of pairwise disjoint curves representing $\lambda([P])$.

The statement is easy to see if $(g, n)=(3,0)$, as there are only three 1 -sided curves in $P$. Since $\lambda$ is injective, there are three curves in $P^{\prime}$. They are all 1 -sided and adjacent to each other with respect to $P^{\prime}$.

Assume that $(g, n) \neq(3,0)$. There exists $x \in P$ such that $a, b, x$ is as in Figure 4(i). Let $a^{\prime} \in \lambda([a]), b^{\prime} \in \lambda([b]), x^{\prime} \in \lambda([x])$ such that $a^{\prime}, b^{\prime}, x^{\prime} \in P^{\prime}$. 


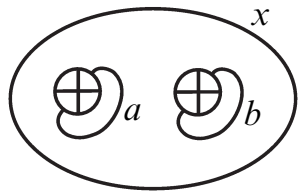

(i)

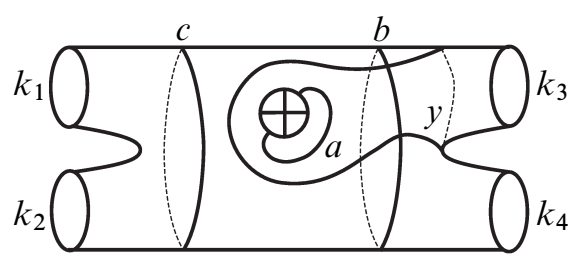

(iv)

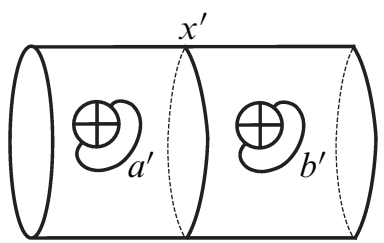

(ii)

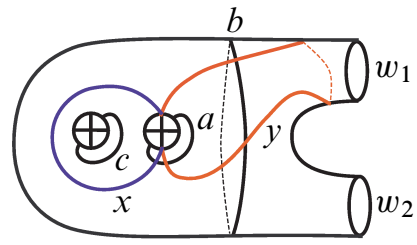

(iii)

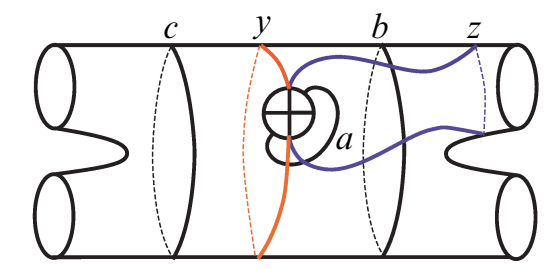

(v)

Figure 4: Curve configurations II

Suppose $g+n \geq 4$ and $(g, n) \neq(2,2)$. There is at least one other curve in $P$ that is on the other side of $x$, and it is adjacent to $x$ with respect to $P$. Since $x$ is a separating curve that has curves that are adjacent to it on both sides, and nonadjacency is preserved by Lemma 2.4, $x^{\prime}$ cannot be a 1-sided curve, otherwise some curves that are not adjacent with respect to $P$ would have to be adjacent with respect to $P^{\prime}$, and this would give a contradiction. By Lemma 2.2, the only curves in a top-dimensional simplex are either 1-sided or separating curves. So, since $x^{\prime}$ is not 1 -sided, $x^{\prime}$ is a separating curve. Since $a, b$ are not adjacent to any curve other than $x$, we see that $a^{\prime}, b^{\prime}$ are not adjacent to any curve other than $x^{\prime}$. This implies that $a^{\prime}, b^{\prime}$ has to be on the same side of $x^{\prime}$, and there should not be any other curve coming from $P^{\prime}$ on that side. This implies that $a^{\prime}$ and $b^{\prime}$ have to be adjacent with respect to $P^{\prime}$.

Suppose $(g, n)=(2,2)$; then by Lemma 2.5, we know that both $a^{\prime}$ and $b^{\prime}$ are 1-sided. Then, $x^{\prime}$ is a separating curve. Suppose $a^{\prime}$ and $b^{\prime}$ are not adjacent with respect to $P^{\prime}$. Then, $a^{\prime}, x^{\prime}, b^{\prime}$ are as shown in Figure 4(ii). This gives a contradiction as $\lambda$ is injective, and there are infinitely many nonisotopic 1 -sided simple closed curves that are disjoint from $x$ on $N$, so their images should have nonisotopic representatives disjoint from $x^{\prime}$, but there are only finitely many nonisotopic simple closed curves on $N$ disjoint from $x^{\prime}$. Hence, $a^{\prime}$ and $b^{\prime}$ have to be adjacent with respect to $P^{\prime}$.

Lemma 2.8 Suppose that $g+n \geq 4$. Let $\lambda: \mathcal{C}(N) \rightarrow \mathcal{C}(N)$ be an injective simplicial map. Let $P$ be a pair of pants decomposition on $N$ that corresponds to a 
top-dimensional maximal simplex in $\mathcal{C}(N)$. Let $a, b \in P$ such that $a$ is 1 -sided, $b$ is 2 -sided and $a$ is adjacent to $b$ with respect to $P$. There exists $a^{\prime} \in \lambda([a])$ and $b^{\prime} \in \lambda([b])$ such that $a^{\prime}$ is adjacent to $b^{\prime}$ with respect to $P^{\prime}$, where $P^{\prime}$ is a set of pairwise disjoint curves representing $\lambda([P])$ containing $a^{\prime}, b^{\prime}$.

Proof Suppose that $g+n \geq 4$. Let $P$ be a pair of pants decomposition on $N$ that corresponds to a top-dimensional maximal simplex in $\mathcal{C}(N)$. Let $a, b \in P$ such that $a$ is 1 -sided, $b$ is 2-sided, and $a$ is adjacent to $b$ with respect to $P$. Let $P^{\prime}$ be a set of pairwise disjoint curves representing $\lambda([P])$. Let $a^{\prime} \in \lambda([a]), b^{\prime} \in \lambda([b])$ such that $a^{\prime}, b^{\prime} \in P^{\prime}$. The statement is easy to see in $(g, n)=(1,3)$ case as there are only two curves in $P$ if $(g, n)=(1,3)$.

Assume that $(g, n) \neq(1,3)$. If $g=1$, then the only 2 -sided curves are separating, so $b$ is a separating curve. If $g \geq 2$, by Lemma $2.2 b$ is a separating curve. So, in all cases $b$ has to be a separating curve. Suppose $a$ is the only curve that is adjacent to $b$ on one side of $b$. Since $g+n \geq 4$ and $(g, n) \neq(1,3)$ there is at least one other curve in $P$ that is on the other side of $b$. Since nonadjacency is preserved, we see that $b^{\prime}$ should be a separating curve, $a^{\prime}$ has to be on one side of $b^{\prime}$, and there should not be any other curve coming from $P^{\prime}$ on that side. This implies that $a^{\prime}$ is adjacent to $b^{\prime}$. In the other cases, by using Lemma 2.2, we see that there exists $c \in P$ such that $a, b, c$ are as shown in Figure 4(iii) or (iv). Let $c^{\prime} \in \lambda([c])$ such that $a^{\prime}, b^{\prime}, c^{\prime}$ have minimal intersection. We note that the curves $w_{1}, w_{2}, k_{1}, k_{2}, k_{3}, k_{4}$ that we see in these figures could be representing boundary components of $N$ or separating curves in $P$ or they could bound Möbius bands depending on the cases we will consider below.

Case 1 Suppose $a, b, c$ are as shown in Figure 4(iii). If $(g, n)=(2,2)$, then by Lemma 2.5 and the previous part, we see that $a^{\prime}, c^{\prime}$ have to be both 1 -sided and adjacent to each other with respect to $P^{\prime}$. This implies that $a^{\prime}, b^{\prime}$ also have to be adjacent to each other with respect to $P^{\prime}$. Suppose $(g, n) \neq(2,2)$. Then $b$ has curves that are adjacent to it on both sides. Since nonadjacency is preserved, $b^{\prime}$ has to be a separating curve, and $a^{\prime}$ and $c^{\prime}$ have to be on the same side of $b^{\prime}$ and there should not be any other curve on that side coming from $P^{\prime}$. Suppose $a^{\prime}$ is not adjacent to $b^{\prime}$. Then, $c^{\prime}$ has to be adjacent to $b^{\prime}$ with respect to $P^{\prime}$. This implies that $a^{\prime}, b^{\prime}, c^{\prime}$ are as shown in Figure 5(i) or (ii). If $a^{\prime}, b^{\prime}, c^{\prime}$ are as shown in Figure 5(i), we get a contradiction by using the curves $x, y$ shown in Figure 4(iii), because $x, y, a$ are pairwise nonisotopic, $x, y$ have small intersection with $a$, and they are both disjoint from $c$. Let $x^{\prime} \in \lambda([x])$ and $y^{\prime} \in \lambda([y])$ such that $a^{\prime}, x^{\prime}, y^{\prime}, c^{\prime}$ have minimal intersection. Since $\lambda$ is injective, $a^{\prime}, x^{\prime}, y^{\prime}, c^{\prime}$ are pairwise nonisotopic. By Lemma 2.3, each of $x^{\prime}$ and $y^{\prime}$ has to intersect $a^{\prime}$ essentially and should be disjoint from $c^{\prime}$; that gives a contradiction. Suppose $a^{\prime}, b^{\prime}, c^{\prime}$ are as shown in Figure 5(ii). We can complete 


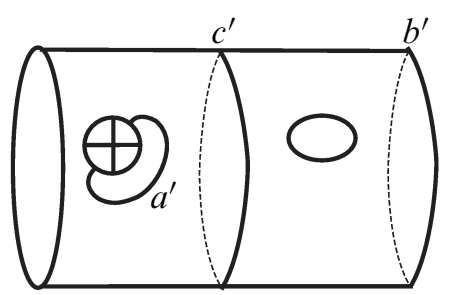

(i)

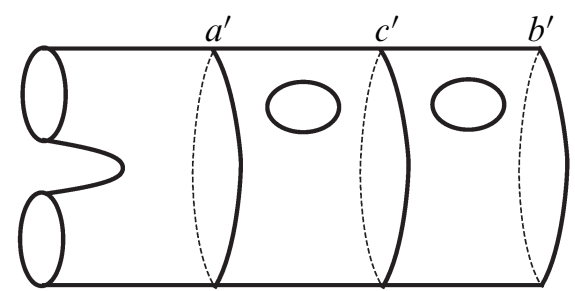

(ii)

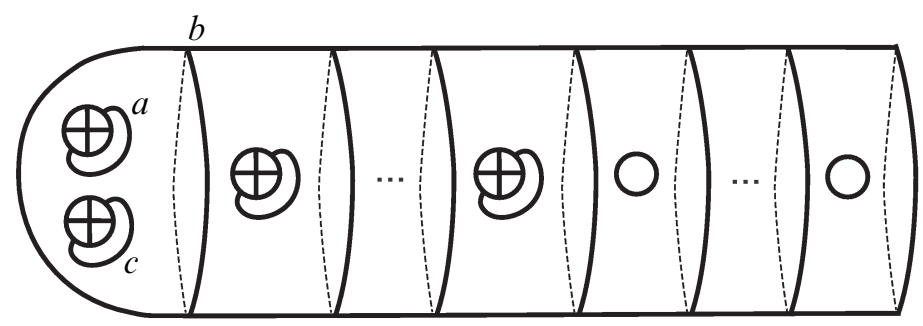

(iii)

Figure 5: Curve configurations III

$a, b, c$ to a top-dimensional pants decomposition $W$ (see Figure 5(iii)), such that there are $g$ 1-sided curves, and the rest are separating curves and there exists at most one separating curve, $v$, in $W$, such that $v$ is not adjacent to any curve in $W$ on one side of it. So, except possibly for one separating curve, separating curves in $W$ are adjacent to at least one curve on both sides with respect to $W$. Since nonadjacency is preserved, the image of all the separating curves in $W$ except possibly the image of $v$ should have separating representatives. This implies that the image of $g$-sided curves in $W$ has to have at least $g-11$-sided curves as representatives, by Lemma 2.2. Hence, in our case in Figure 5(ii), we get a contradiction because both $a^{\prime}$ and $c^{\prime}$ cannot be separating curves at the same time, as $a$ and $c$ are 1 -sided curves. Hence, $a^{\prime}$ has to be adjacent to $b^{\prime}$.

Case 2 Suppose $a, b, c$ are as shown in Figure 4(iv). Suppose there is a curve, $x \in P$, on the side of $b$ that does not contain $a$. By using Lemma 2.2, we see that $b^{\prime}$ is a separating curve as nonadjacency is preserved and $b$ has curves that are adjacent to it on both sides, and $a^{\prime}$ and $c^{\prime}$ are on the same side of $b^{\prime}$. Since $a$ is not adjacent to any curve other than $b$ and $c$, we see that $a^{\prime}$ will not be adjacent to any curve other than $b^{\prime}$ and $c^{\prime}$. If there is a third curve of $P$ that is on the same side of $b$ as $a$ and $c$, that will imply that $a^{\prime}$ has to be adjacent to $b^{\prime}$ since nonadjacency is preserved. Suppose there is no other curve of $P$ on the side of $b$ that contains $a$ and $c$. We will see that 


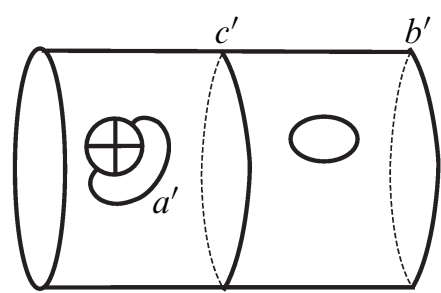

(i)

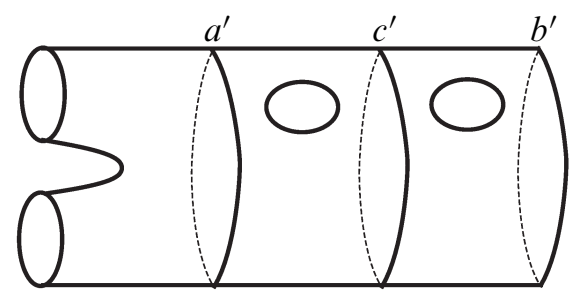

(ii)

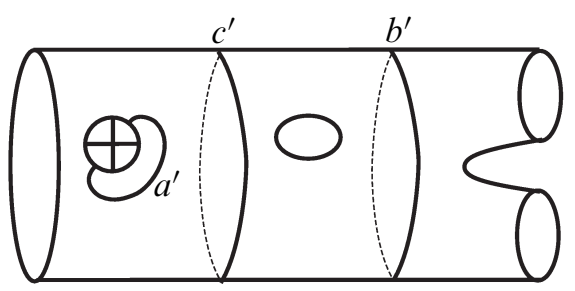

(iii)

Figure 6: Curve configurations IV

$a^{\prime}$ is adjacent to $b^{\prime}$ with respect to $P^{\prime}$ as follows: Suppose $a^{\prime}$ is not adjacent to $b^{\prime}$ with respect to $P^{\prime}$. Then $c^{\prime}$ has to be adjacent to $b^{\prime}$ and $a^{\prime}$, and $a^{\prime}, b^{\prime}, c^{\prime}$ have to be as shown in Figure 6(i) or (ii). In both cases we get a contradiction as follows: By changing the curve $b$ to $y$ as shown in Figure 4(iv) we get another top-dimensional pants decomposition, say $W$, such that $W=(P \backslash\{b\}) \cup\{y\}$. We see that $y$ only has small intersection with $b$, and $y$ is disjoint from all the other curves in $P$, and $a$ and $c$ are not adjacent with respect to $W$. But if $a^{\prime}, b^{\prime}, c^{\prime}$ are as shown in Figure 6(i) or (ii), $a^{\prime}$ and $c^{\prime}$ have to be adjacent to each other with respect to $W^{\prime}$; that gives a contradiction. Hence, $a^{\prime}$ is adjacent to $b^{\prime}$ with respect to $P^{\prime}$.

Suppose there is a curve in $P$ on the side of $c$ that does not contain $a$, and the side of $b$ that does not contain $a$ does not have any curves from $P$. Then, by using that nonadjacency is preserved we see that $c^{\prime}$ is a separating curve, $a^{\prime}, b^{\prime}$ are on the same side of $c^{\prime}$, and there are not any other curves of $P^{\prime}$ on the side of $c^{\prime}$ containing $a^{\prime}, b^{\prime}$. This implies that $a^{\prime}$ and $b^{\prime}$ are adjacent with respect to $P^{\prime}$.

Suppose the sides of each of $b$ and $c$ that do not contain $a$ do not have any essential curves in $P$. Then we have $(g, n)=(1,4)$, and $a, b, c$ are as shown in Figure 4(v). In this case, we know that $a^{\prime}$ is 1 -sided and $b^{\prime}, c^{\prime}$ are separating curves by Lemma 2.5. Suppose $a^{\prime}$ and $b^{\prime}$ are not adjacent with respect to $P^{\prime}$. Then, $a^{\prime}, b^{\prime}, c^{\prime}$ are as shown Figure 6(iii). In such a case, by considering curves $z, y$ given in Figure 4(v) we get a contradiction as follows: $a, c, y, z$ are pairwise nonisotopic and each of $y, z$ has 


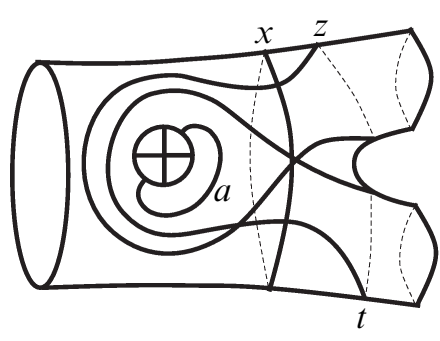

(i)

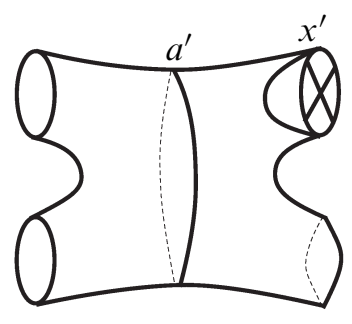

(ii)

Figure 7: Curve configurations V

small intersection with $a$, and they are disjoint from $c$. Let $y^{\prime}, z^{\prime}$ be representatives of $\lambda([y]), \lambda([z])$ respectively such that $a^{\prime}, c^{\prime}, y^{\prime}, z^{\prime}$ have minimal intersection. Each of $a^{\prime}, c^{\prime}, y^{\prime}, z^{\prime}$ are pairwise nonisotopic and each of $y^{\prime}, z^{\prime}$ intersects $a^{\prime}$, and is disjoint from $c^{\prime}$. This gives a contradiction. So, $a^{\prime}$ is adjacent to $b^{\prime}$ with respect to $P^{\prime}$.

By Lemma 2.2, the curves in a pants decomposition that corresponds to a top-dimensional simplex in $\mathcal{C}(N)$ are either separating or 1-sided with nonorientable complement. So, combining our results in Lemmas 2.6, 2.7 and 2.8, we get the following:

Lemma 2.9 Suppose that $(g, n)=(3,0)$ or $g+n \geq 4$. Let $\lambda: \mathcal{C}(N) \rightarrow \mathcal{C}(N)$ be an injective simplicial map. Let $P$ be a pair of pants decomposition on $N$ that corresponds to a top-dimensional maximal simplex in $\mathcal{C}(N)$. Let $a, b \in P$ such that $a$ is adjacent to $b$ with respect to $P$. There exists $a^{\prime} \in \lambda([a])$ and $b^{\prime} \in \lambda([b])$ such that $a^{\prime}$ is adjacent to $b^{\prime}$ with respect to $P^{\prime}$, where $P^{\prime}$ is a set of pairwise disjoint curves representing $\lambda([P])$ containing $a^{\prime}, b^{\prime}$.

Lemma 2.10 Suppose that $g=1$ and $n \geq 3$. Let $\lambda: \mathcal{C}(N) \rightarrow \mathcal{C}(N)$ be an injective simplicial map. If $a$ is a 1 -sided simple closed curve on $N$, then $\lambda([a])$ is the isotopy class of a 1-sided simple closed curve on $N$.

Proof Suppose that $g=1$ and $n \geq 3$. Let $a$ be a 1 -sided simple closed curve on $N$. Since genus is 1 , the complement of $a$ is orientable. Let $a^{\prime} \in \lambda([a])$.

Suppose $a^{\prime}$ is a 2 -sided separating simple closed curve. If $n=3$, then we complete $a$ to a pair of pants decomposition $P=\{a, x\}$ as shown in Figure 7(i). Let $P^{\prime}$ be a set of pairwise disjoint elements of $\lambda([P])$ containing $a^{\prime}$, and let $x^{\prime} \in \lambda([x]) \cap P^{\prime}$. Since $a^{\prime}$ is separating, $x^{\prime}$ has to be a 1 -sided curve as shown in Figure 7(ii). Let $z$ and $t$ be as shown in Figure 7(i). We see that $a, x, z, t$ are pairwise nonisotopic, each of $z$ and 
$t$ has small intersection with $x$, and each of them is disjoint from $a$. Let $z^{\prime} \in \lambda([z])$, $t^{\prime} \in \lambda([t])$ such that $z^{\prime}, t^{\prime}, a^{\prime}, x^{\prime}$ intersect minimally. Since $\lambda$ is injective, $a^{\prime}, x^{\prime}, z^{\prime}, t^{\prime}$ are pairwise nonisotopic. By using Lemma 2.3, we see that each of $z^{\prime}, t^{\prime}$ is disjoint from $a^{\prime}$, and intersects $x^{\prime}$ essentially. So, $z^{\prime}, t^{\prime}$ both have to lie in the subsurface, $N_{1}$, a projective plane with two boundary components that contains $x^{\prime}$ and has $a^{\prime}$ as a boundary component as shown in Figure 7(ii). This gives a contradiction, since in $N_{1}$ there are no two nontrivial, nonisotopic simple closed curves that intersect $x^{\prime}$ essentially; see Scharlemann's result in [30].

If $n \geq 4$, then we complete $a$ to a pair of pants decomposition $P=\left\{a, x_{1}, \ldots, x_{n-2}\right\}$ as shown in Figure 8(i). $P$ corresponds to a top-dimensional maximal simplex. Let $P^{\prime}$ be a set of pairwise disjoint curves representing $\lambda([P])$ containing $a^{\prime}$. Let $x_{i}^{\prime} \in \lambda\left(\left[x_{i}\right]\right) \cap P^{\prime}$ for each $i=1,2, \ldots, n-2$. By Lemma 2.9 and Lemma $2.4, \lambda$ preserves adjacency and nonadjacency with respect to top-dimensional maximal simplices. By using that adjacency and nonadjacency are preserved, $n \geq 4$ and $g=1$, we see that the elements of $P^{\prime}$ should be as shown in Figure 8(ii). Consider the curves $z, t$ given in Figure 8(i). We see that $\left[x_{n-3}\right],\left[x_{n-2}\right],[z],[t]$ are all distinct elements and each of $z$ and $t$ have small intersection with $x_{n-2}$, and each of them is disjoint from $x_{n-3}$. Let $z^{\prime} \in \lambda([z])$, $t^{\prime} \in \lambda([t])$ such that each of $z^{\prime}$ and $t^{\prime}$ intersects each of $x_{n-3}^{\prime}, x_{n-2}^{\prime}$ minimally. Since $\lambda$ is injective, $\left[x_{n-3}^{\prime}\right],\left[x_{n-2}^{\prime}\right],\left[z^{\prime}\right],\left[t^{\prime}\right]$ are all distinct elements. By using Lemma 2.3, we see that each of $z^{\prime}, t^{\prime}$ is disjoint from $x_{n-3}^{\prime}$, and intersects $x_{n-2}^{\prime}$ essentially. So, $z^{\prime}$ and $t^{\prime}$ both have to lie in the subsurface, $N_{1}$, which is a projective plane with two boundary components containing $x_{n-2}^{\prime}$, and having $x_{n-3}^{\prime}$ as a boundary component as shown in Figure 8(ii). This gives a contradiction as before. So, $a^{\prime}$ cannot be a 2 -sided separating simple closed curve. Since $g=1, a^{\prime}$ cannot be a 2 -sided nonseparating simple closed curve. Hence, $a^{\prime}$ is a 1 -sided simple closed curve on $N$.

Lemma 2.11 Suppose that $g=2$ and $n \geq 2$. Let $\lambda: \mathcal{C}(N) \rightarrow \mathcal{C}(N)$ be an injective simplicial map. If $a$ is a 1-sided simple closed curve on $N$ whose complement is nonorientable, then $\lambda([a])$ is not the isotopy class of a separating simple closed curve on $N$.

Proof Let $a$ be a 1 -sided simple closed curve on $N$ whose complement is nonorientable. Let $a^{\prime} \in \lambda([a])$. Suppose $a^{\prime}$ is a separating simple closed curve on $N$.

We complete $a$ to a curve configuration $a, x$ as shown in Figure 9(i), using a boundary component $\partial_{1}$ of $N$. Let $T$ be the subsurface of $N$ having $x$ and $\partial_{1}$ as its boundary as shown in Figure 9(i). $T$ is a projective plane with two boundary components. The curves $a$ and $x$ can be completed to a pair of pants decomposition $P$ on $N$ that corresponds to a top-dimensional maximal simplex in $\mathcal{C}(N)$. Let $P^{\prime}$ be a set of 


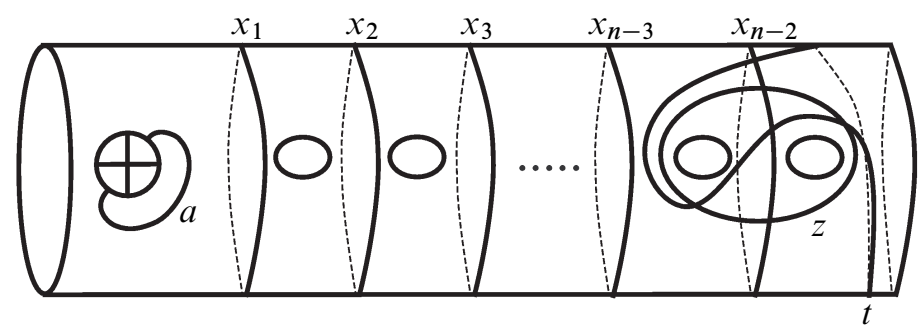

(i)

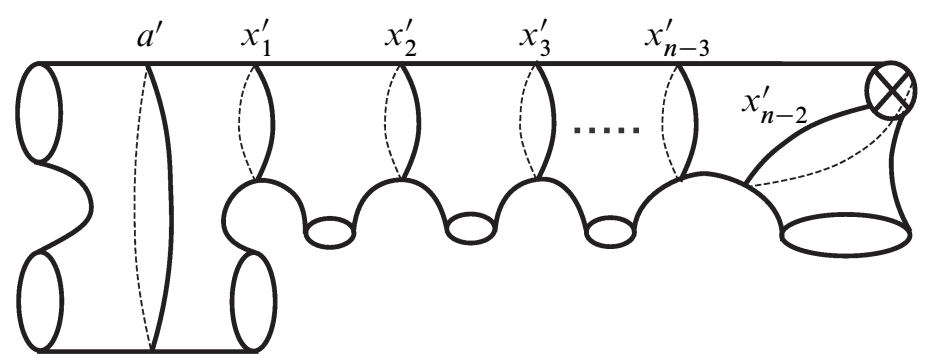

(ii)

Figure 8: Curve configurations VI

pairwise disjoint curves representing $\lambda([P])$ containing $a^{\prime}$, and let $x^{\prime} \in \lambda([x]) \cap P^{\prime}$. By Lemma 2.9 and Lemma 2.4 we know that $\lambda$ preserves adjacency and nonadjacency with respect to top-dimensional maximal simplices. Since $a$ is adjacent to only $x$ with respect to $P$, the curve $a^{\prime}$ is adjacent to only $x^{\prime}$ with respect to $P^{\prime}$. Since $g=2$ and $n \geq 2$, there is a curve $z$ in $P$ such that $x$ is adjacent to $z$ with respect to $P$ and $a$ is different from $z$. Since $a^{\prime}$ is a separating curve and $a^{\prime}$ is only adjacent to $x^{\prime}$ with respect to $P^{\prime}$, and $x^{\prime}$ is adjacent to at least two curves with respect to $P^{\prime}$, we see that there is a four holed sphere, $R_{1}$, having $x^{\prime}$ and three boundary components of $N$ (if they exist) as its boundary components such that $a^{\prime}$ divides $R_{1}$ into two pair of pants as shown in Figure 9(iii). Hence, if $(g, n)=(2,2)$ we get a contradiction as there are not enough boundary components.

Suppose $g=2, n \geq 3$. We complete $a$ to a pair of pants decomposition $Q=$ $\left\{a, b, x_{1}, \ldots, x_{n-1}\right\}$ that corresponds to a top-dimensional maximal simplex as shown in Figure 9(ii). In the figure we see that $a$ is adjacent to only $b$ and $x_{1}$ with respect to $Q$. Let $Q^{\prime}$ be a set of pairwise disjoint curves representing $\lambda([Q])$ containing $a^{\prime}$. Let

$$
b^{\prime} \in \lambda([b]) \cap Q^{\prime}, \quad x_{i}^{\prime} \in \lambda\left(\left[x_{i}\right]\right) \cap Q^{\prime} \quad \text { for } i=1,2, \ldots, n-1 .
$$

By using that $a^{\prime}$ is separating and $\lambda$ preserves adjacency and nonadjacency with respect to $Q$, we see that $Q^{\prime}$ is as shown in Figure 9(iv). By choosing curves $z, t$ as shown in 


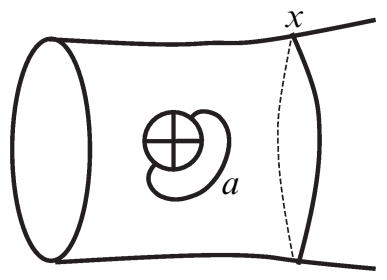

(i)

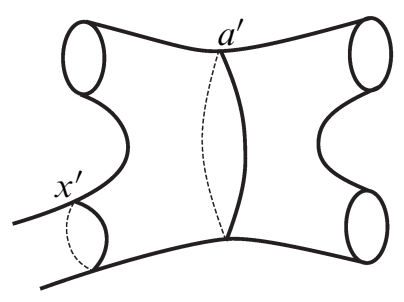

(iii)

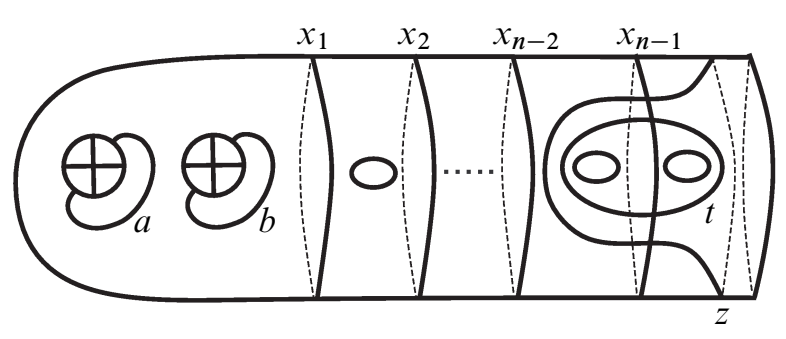

(ii)

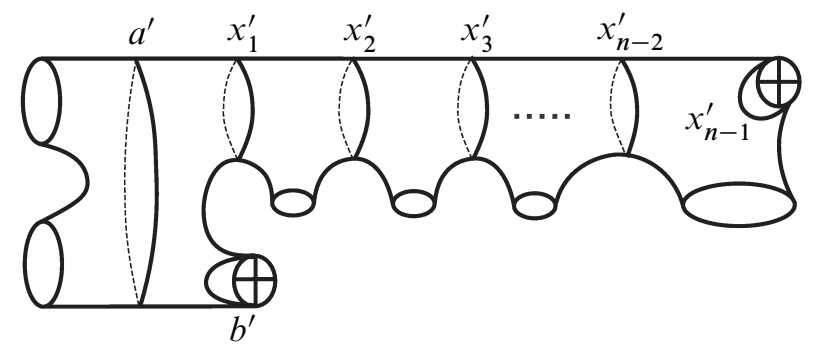

(iv)

Figure 9: Curve configurations VII

Figure 9(ii) we get a contradiction as in Lemma 2.10. Hence, $a^{\prime}$ cannot be a separating simple closed curve.

Lemma 2.12 Suppose that $g \geq 3$. Let $\lambda: \mathcal{C}(N) \rightarrow \mathcal{C}(N)$ be an injective simplicial map. If $a$ is a 1-sided simple closed curve on $N$ whose complement is nonorientable, then $\lambda([a])$ is not the isotopy class of a separating simple closed curve on $N$.

Proof Let $a$ be a 1 -sided simple closed curve on $N$ whose complement is nonorientable. Let $a^{\prime} \in \lambda([a])$. If $(g, n)=(3,0)$ then there is no nontrivial separating simple closed curve on $N$. So, the result follows.

Assume that $a^{\prime}$ is a separating simple closed curve on $N$. We will consider the following cases:

(a) If $n=0$ and $g \geq 4$, then we complete $a$ to a pants decomposition $P$ such that $P$ corresponds to a top-dimensional maximal simplex in $\mathcal{C}(N)$, and $a$ is adjacent to only two curves with respect to $P$ (see Figure 10(i)). Let $P^{\prime}$ be a set of pairwise disjoint curves representing $\lambda([P])$ containing $a^{\prime}$. Since $\lambda$ is injective, $P^{\prime}$ also corresponds to a top-dimensional maximal simplex. Since adjacency and nonadjacency with respect to top-dimensional maximal simplices are preserved by Lemma 2.9 and Lemma 2.4, 


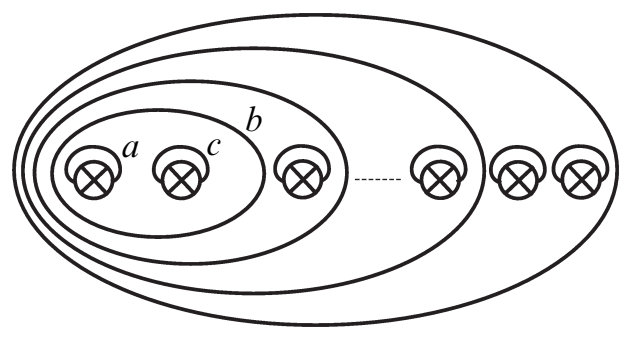

(i)

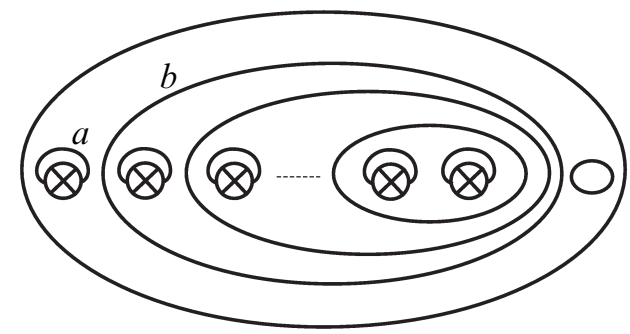

(ii)

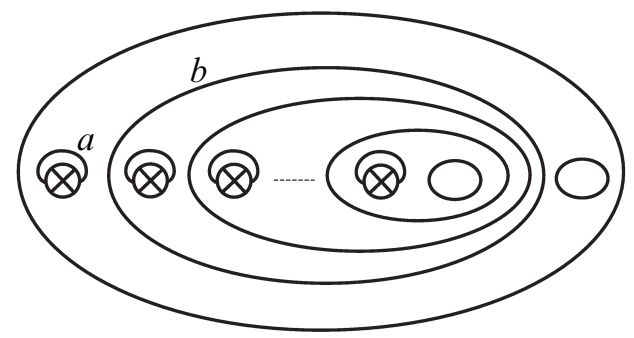

(iii)

Figure 10: Pants decompositions

we see that $a^{\prime}$ has to be adjacent to only two simple closed curves with respect to $P^{\prime}$. This is impossible since $a^{\prime}$ is a separating curve, $n=0$, and $g \geq 4$. So, we get a contradiction.

(b) If $n=1$ or $n=2$, and $g \geq 3$, then we complete $a$ to a pair of pants decomposition $P$ such that $P$ corresponds to a top-dimensional maximal simplex in $\mathcal{C}(N)$, and $a$ is adjacent to only one curve with respect to $P$ as shown in Figure 10(ii) and Figure 10(iii). Since adjacency and nonadjacency with respect to top-dimensional maximal simplices are preserved by Lemma 2.9 and Lemma 2.4, we see that $a^{\prime}$ has to be adjacent to only one simple closed curve in the image pants decomposition that corresponds to a top-dimensional maximal simplex. This is impossible since $a^{\prime}$ is separating, $g \geq 3$ and there is only one or two boundary components. So, we get a contradiction.

(c) If $n \geq 3$ and $g \geq 3$, then we get a contradiction as follows: If $g=3$, then we complete $a$ to a pair of pants decomposition $P=\left\{a, b, c, d, x_{1}, \ldots, x_{n-1}\right\}$ as shown in Figure 11(i). We see that $P$ corresponds to a top-dimensional maximal simplex in $\mathcal{C}(N)$. Let $P^{\prime}$ be a set of pairwise disjoint curves representing $\lambda([P])$ containing $a^{\prime}$. The set $P^{\prime}$ also corresponds to a top-dimensional maximal simplex in $\mathcal{C}(N)$. Let $b^{\prime} \in \lambda([b]) \cap P^{\prime}, c^{\prime} \in \lambda([c]) \cap P^{\prime}, d^{\prime} \in \lambda([d]) \cap P^{\prime}$, and $x_{i}^{\prime} \in \lambda\left(\left[x_{i}\right]\right) \cap P^{\prime}$ for $i=1, \ldots, n-1$. By using that adjacency and nonadjacency are preserved with respect to top-dimensional maximal simplices, we get $P^{\prime}=\left\{a^{\prime}, b^{\prime}, c^{\prime}, d^{\prime}, x_{1}^{\prime}, \ldots, x_{n-1}^{\prime}\right\}$ as 


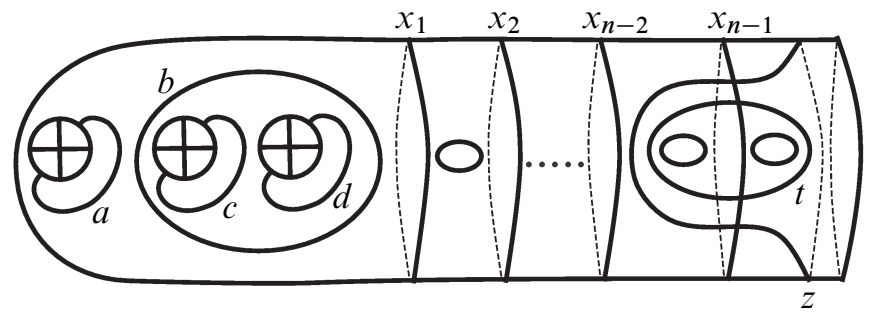

(i)

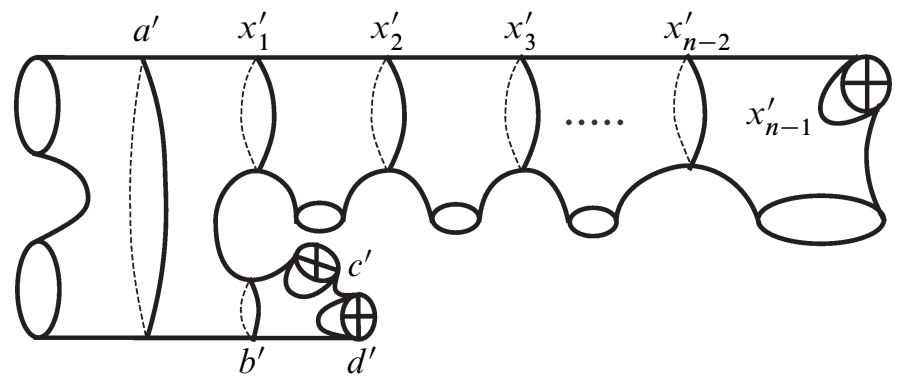

(ii)

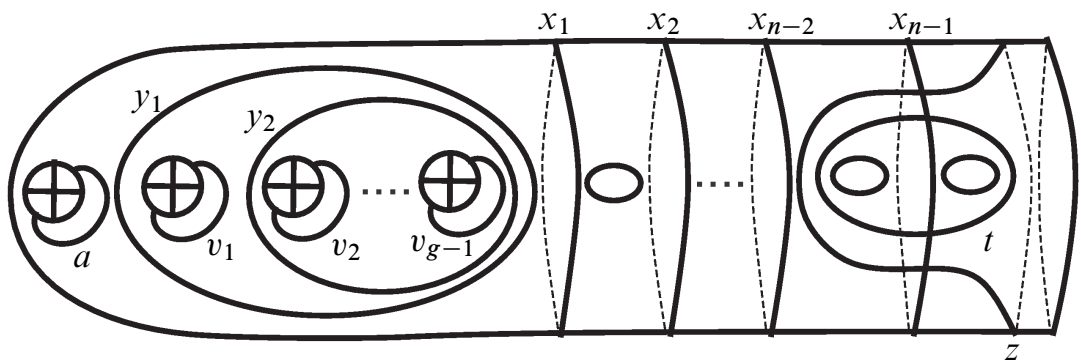

(iii)

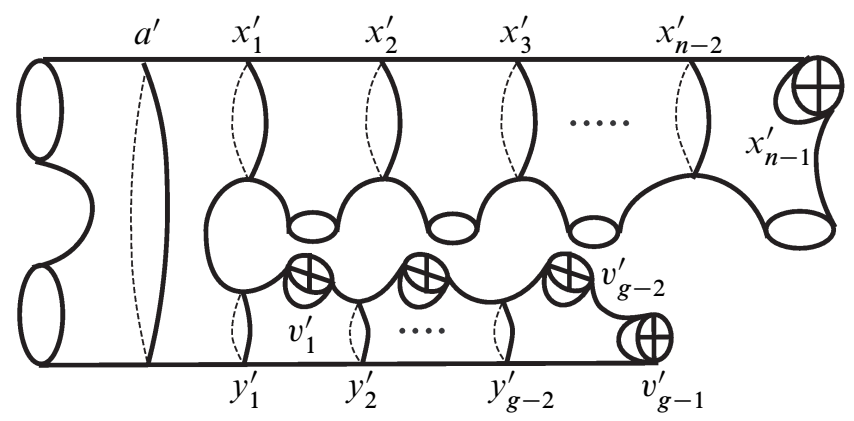

(iv)

Figure 11: Curve configurations VIII 
shown in Figure 11(ii). Let $z$ and $t$ be as shown in Figure 11(i). By using the curves $z, t$, we get a contradiction as in the proof of Lemma 2.11. Similarly, if $g \geq 4$, we complete $a$ to a pair of pants decomposition $P$ that corresponds to a top-dimensional maximal simplex as shown in Figure 11(iii), and we get the corresponding pair of pants decomposition as shown in Figure 11(iv). Using the curves $z, t$ shown in Figure 11(iii), we get a contradiction as in the proof of Lemma 2.11.

We proved that $a^{\prime}$ cannot be separating simple closed curve.

Lemma 2.13 Let $g \geq 2$. Suppose that $(g, n)=(3,0)$ or $g+n \geq 4$. Let

$$
\lambda: \mathcal{C}(N) \rightarrow \mathcal{C}(N)
$$

be an injective simplicial map. If $a$ is a 1-sided simple closed curve on $N$ whose complement is nonorientable, then $\lambda([a])$ is the isotopy class of a 1-sided simple closed curve whose complement is nonorientable.

Proof Let $a$ be a 1-sided simple closed curve whose complement is nonorientable. Let $a^{\prime} \in \lambda([a])$. By Lemma 2.11 and Lemma 2.12, $a^{\prime}$ cannot be a separating simple closed curve. Since $a$ is a 1 -sided simple closed curve whose complement is nonorientable, $[a]$ is the vertex of a top-dimensional maximal simplex in $\mathcal{C}(N)$. Since $\lambda$ is injective, the image of this simplex is a top-dimensional maximal simplex containing $\left[a^{\prime}\right]$. If $a^{\prime}$ is a 1 -sided simple closed curve whose complement is orientable or a 2-sided nonseparating simple closed curve, then $\left[a^{\prime}\right]$ cannot be a vertex of a top-dimensional maximal simplex in $\mathcal{C}(N)$ by Lemma 2.2. Hence, $a^{\prime}$ is a 1-sided simple closed curve whose complement is nonorientable.

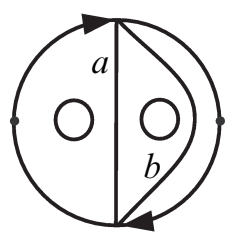

(i)
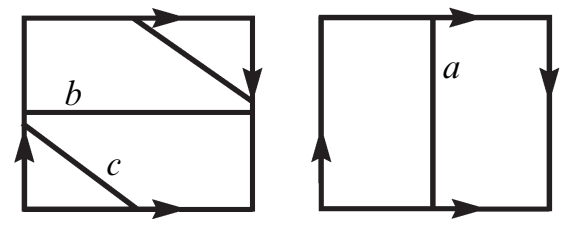

(ii)

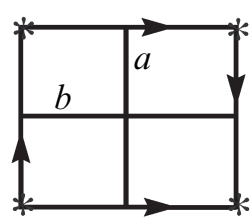

(iii)

Figure 12: Some curves when (i) $(g, n)=(1,2)$, (ii) $(g, n)=(2,0)$, (iii) $(g, n)=(2,1)$

The following theorem is given by the author in [14]. 


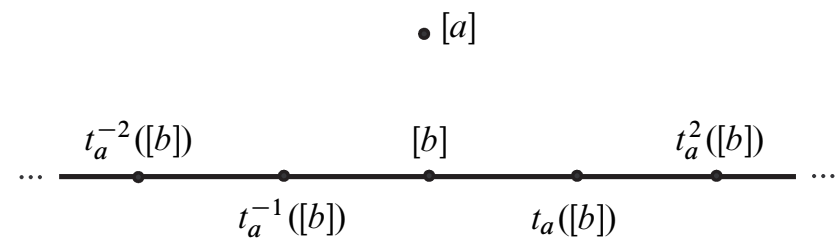

(i)

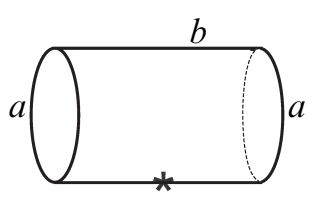

(ii)

Figure 13: Curve complex for $(g, n)=(2,1)$

Theorem 2.14 Let $N$ be a compact, connected, nonorientable surface of genus $g$ with $n$ boundary components. Suppose that either $(g, n) \in\{(1,0),(1,1),(2,0),(2,1),(3,0)\}$ or $g+n \geq 5$. If $\lambda: \mathcal{C}(N) \rightarrow \mathcal{C}(N)$ is a superinjective simplicial map, then $\lambda$ is induced by a homeomorphism $h: N \rightarrow N$.

Now we state our result:

Theorem 2.15 Let $N$ be a compact, connected, nonorientable surface of genus $g$ with $n$ boundary components. Suppose that $g+n \leq 3$ or $g+n \geq 5$. If $\lambda: \mathcal{C}(N) \rightarrow \mathcal{C}(N)$ is an injective simplicial map, then $\lambda$ is induced by a homeomorphism $h: N \rightarrow N$.

Proof Let $\lambda: \mathcal{C}(N) \rightarrow \mathcal{C}(N)$ be an injective simplicial map. If $(g, n)=(1,0)$, then $N$ is the projective plane. If $(g, n)=(1,1)$, then $N$ is Möbius band. In both cases there is only one vertex, the isotopy class of a 1 -sided curve, in $\mathcal{C}(N)$. So, $\lambda$ is induced by the identity homeomorphism in both cases. If $(g, n)=(1,2)$, then there are only two vertices (the isotopy classes of the curves $a$ and $b$ as shown in Figure 12(i)) in $\mathcal{C}(N)$ (see [30]). If $\lambda$ fixes each of the vertices, then it is induced by the identity homeomorphism. If $\lambda$ switches them, then it is induced by a homeomorphism that switches $a$ and $b$.

If $(g, n)=(2,0)$, then there are only three vertices and there is only one edge in $\mathcal{C}(N)$ (see [30]). The vertices are the isotopy classes of the curves $a, b$ and $c$ as shown in Figure 12(ii). The curves $b$ and $c$ are both 1 -sided, and $a$ is 2 -sided. The vertices $[b]$ and $[c]$ are connected by an edge. Since $\lambda$ is an injective simplicial map, it sends vertices connected by an edge to vertices connected by an edge. So, it sends $\{[b],[c]\}$ to $\{[b],[c]\}$ and fixes $[a]$. If $\lambda$ fixes each of $[b]$ and $[c]$, then it is induced by the identity homeomorphism. If $\lambda$ switches $[b]$ and $[c]$, then it is induced by a homeomorphism that switches $b$ and $c$, and fixes $a$ up to isotopy. 
If $(g, n)=(2,1)$, then $\mathcal{C}(N)$ is given by Scharlemann in [30] as follows: Let $a$ and $b$ be the curves shown in Figure 12(iii) (in the picture we draw a puncture instead of the boundary component). Let $t_{a}$ be the Dehn twist about the 2-sided curve $a$. The vertex set of $\mathcal{C}(N)$ is $\left\{[a], t_{a}^{m}([b]): m \in \mathbb{Z}\right\}$. The complex is shown in Figure 13(i). Each element in $\left\{t_{a}^{m}([b]): m \in \mathbb{Z}\right\}$ is connected to two elements in that set. Since $\lambda$ is an injective simplicial map, it sends vertices connected by an edge to vertices connected by an edge. So, $\lambda$ fixes $[a]$ and sends $\left\{t_{a}^{m}([b]): m \in \mathbb{Z}\right\}$ to itself. By cutting $N$ along $a$ we get a cylinder with one puncture as shown in Figure 13(ii). There is a reflection of this cylinder with one puncture, which interchanges the front face with the back face and fixes $b$ pointwise. This gives a homeomorphism $l$ of $N$ such that $l(b)=b$ and $l(a)=a^{-1}$. Let $l_{\#}$ be the automorphism of $\mathcal{C}(N)$ induced by $l$. We have $l_{\#}([b])=[b]$ and $l_{\#}\left(t_{a}([b])\right)=t_{a}{ }^{-1}([b])$. The map $l_{\#}$ reflects the graph in Figure 13(i) at $[b]$ and fixes the vertex $[a]$. It is easy to see that $\lambda$ is induced by $t_{a}^{m}$ or $t_{a}^{m} \circ l$ for some $m \in \mathbb{Z}$. This completes the proof of our theorem for $g+n \leq 3$ except for $(g, n)=(3,0)$ case.

For the remaining cases when $(g, n)=(3,0)$ or $g+n \geq 5$, we will refer to the proofs of Theorems 4.3, 4.4, 4.5, 4.6 in [14]. In these theorems in [14] the author proved that each superinjective simplicial map is induced by a homeomorphism for different cases. We stated the combined main result of [14] as Theorem 2.14 in this paper. The proofs of Theorems 4.3, 4.4, 4.5, 4.6 in [14] use some known results and they are based on some properties of superinjective simplicial maps. We will write these properties of superinjective simplicial maps below and compare them to the properties of our injective simplicial map $\lambda$.

By [14, Lemma 3.2], a superinjective simplicial map is injective. We know $\lambda$ is injective. By [14, Lemma 3.3], a superinjective simplicial map preserves adjacency when $(g, n)=(3,0)$ or $g+n \geq 4$. We obtained this property for $\lambda$ in Lemma 2.9 in this paper. By [14, Lemma 3.4], a superinjective simplicial map preserves nonadjacency when $g+n \geq 4$. We obtained this property for $\lambda$ in Lemma 2.4 in this paper. By [14, Lemma 2.1], if $a$ is a 1-sided simple closed curve on $N$, then a superinjective simplicial map sends $[a]$ to the isotopy class of a 1-sided simple closed curve on $N$ (this result was used for the proof in $g=1$ case). We obtained this property for $\lambda$ when $g=1$ and $n \geq 3$ in Lemma 2.10 in this paper. By [14, Lemma 3.5], if $a$ is a 1-sided simple closed curve on $N$ whose complement is nonorientable, then a superinjective simplicial map sends $[a]$ to the isotopy class of a 1-sided simple closed curve on $N$ whose complement is nonorientable when $(g, n)=(3,0)$ or $g+n \geq 4$. We obtained this property for $\lambda$ in Lemma 2.13 in this paper. By [14, Lemma 2.1], a superinjective simplicial map sends the isotopy class of a 2 -sided curve to the isotopy class of a 2 -sided curve. We do not know this for $\lambda$ in general, but this will not matter, because this property was used only in very special cases in the proofs of Theorems 4.3, 4.4, 
4.5, 4.6 in [14]. The argument can be made easily for those special cases in a different way for $\lambda$ anyway. We will make a point of it below whenever needed. We note that finding all of these properties for injective maps was harder.

By replacing the word superinjective with injective, using all the properties of $\lambda$ that we listed above and making only minor changes in two places in the proofs of Theorems $4.3,4.4,4.5,4.6$ in [14], we get our proofs when $(g, n)=(3,0)$ or $g+n \geq 5$. Instead of rewriting the long proofs of these theorems with these little changes in this paper, we will refer to the proofs of these theorems as follows: When $g=1$ and $n \geq 4$, the proof of our theorem follows by following the proof of Theorem 4.3 in [14], by replacing the word superinjective with injective, using that if $a$ is a 1-sided simple closed curve on $N$ then $\lambda([a])$ is the isotopy class of a 1-sided simple closed curve on $N, \lambda$ preserves adjacency and nonadjacency, and noting that at the point of the usage of the statement of Lemma 2.1 in [14], the argument can be seen easily because the genus is one. When $g=2$ and $n \geq 3$, the proof of our theorem follows by following the proof of Theorem 4.4 in [14], by replacing the word superinjective with injective, using that if $a$ is a 1 -sided simple closed curve on $N$ whose complement is nonorientable then $\lambda([a])$ is the isotopy class of a 1-sided simple closed curve whose complement is nonorientable, $\lambda$ preserves adjacency and nonadjacency, and noting that when there is a referral to Lemma 2.1 in [14], the argument can be seen by considering our result for $(g, n)=(2,1)$ case that we gave above. When $g \geq 3, g+n \geq 5$, the proof of our theorem follows by following the proof of Theorem 4.5 in [14], by replacing the word superinjective with injective, and using the properties of $\lambda$ that we listed. Similarly, when $(g, n)=(3,0)$ the proof of our theorem follows by following the proof of Theorem 4.6 in [14]. This covers all the cases when $(g, n)=(3,0)$ or $g+n \geq 5$, which completes our proof.

\section{Connectivity property}

In this section we will give two different proofs for the following statement: If $\lambda: \mathcal{C}(N) \rightarrow \mathcal{C}(N)$ is a simplicial map that satisfies the connectivity property, then $\lambda$ is induced by a homeomorphism $h: N \rightarrow N$ in most cases.

We say that a simplicial map $\lambda: \mathcal{C}(N) \rightarrow \mathcal{C}(N)$ satisfies the connectivity property if the map satisfies the following: $[a]$ and $[b]$ are connected by an edge in $\mathcal{C}(N)$ if and only if $\lambda([a])$ and $\lambda([b])$ are connected by an edge in $\mathcal{C}(N)$ for every pair of vertices $[a],[b]$ in $\mathcal{C}(N)$.

We remind that a simplicial map $\tau: \mathcal{C}(N) \rightarrow \mathcal{C}(N)$ is called superinjective if it satisfies the following condition: If $[a],[b]$ are two vertices in $\mathcal{C}(N)$, then $i([a],[b])=0$ if and only if $i(\tau([a]), \tau([b]))=0$. 
We note that these two definitions are equivalent on orientable surfaces. On nonorientable surfaces, if $a$ is a 1 -sided simple closed curve, then $i([a],[a])=1$. Hence, a superinjective map on a nonorientable surface sends the isotopy class of a 1-sided curve to the isotopy class of a 1-sided curve, and it sends the isotopy class of a 2 -sided curve to the isotopy class of a 2-sided curve by definition. But a map that satisfies the connectivity property on a nonorientable surface does not have to do this by definition.

Lemma 3.1 Let $(g, n)=(3,0)$ or $g+n \geq 4$. If $\tau: \mathcal{C}(N) \rightarrow \mathcal{C}(N)$ is a superinjective simplicial map, then $\tau$ satisfies the connectivity property.

Proof Let $\tau: \mathcal{C}(N) \rightarrow \mathcal{C}(N)$ be a superinjective simplicial map. By [14, Lemma 3.2], $\tau$ is injective. Let $[a],[b]$ be two vertices in $\mathcal{C}(N)$. We have the following: $[a]$ and $[b]$ are connected by an edge in $\mathcal{C}(N)$ if and only if $(i([a],[b])=0$ and $[a] \neq[b])$ if and only if $(i(\tau([a]), \tau([b]))=0$ and $\tau([a]) \neq \tau([b]))$ if and only if $\tau([a])$ and $\tau([b])$ are connected by an edge in $\mathcal{C}(N)$.

Now we prove some properties of simplicial maps that satisfy the connectivity property:

Lemma 3.2 Let $\lambda: \mathcal{C}(N) \rightarrow \mathcal{C}(N)$ be a simplicial map that satisfies the connectivity property. If $(g, n)=(3,0)$ or $g+n \geq 4$, then $\lambda$ is injective.

Proof Let $[a]$ and $[b]$ be two distinct vertices in $\mathcal{C}(N)$. If $[a]$ and $[b]$ are connected by an edge, then $\lambda([a])$ and $\lambda([b])$ are connected by an edge. So, $\lambda([a]) \neq \lambda([b])$. If $[a]$ and $[b]$ are not connected by an edge, then we can find a simple closed curve $c$ such that $[a]$ and $[c]$ are connected by an edge, and $[b]$ and $[c]$ are not connected by an edge. Then we will have that $\lambda([a])$ and $\lambda([c])$ are connected by an edge, and $\lambda([b])$ and $\lambda([c])$ are not connected by an edge. This gives us that $\lambda([a]) \neq \lambda([b])$. Hence, $\lambda$ is injective.

Lemma 3.3 Let $\lambda: \mathcal{C}(N) \rightarrow \mathcal{C}(N)$ be a simplicial map that satisfies the connectivity property. Let $(g, n)=(3,0)$ or $g+n \geq 4$. If $a$ and $b$ are two nonisotopic simple closed curves on $N$, then $i([a],[b])=0$ if and only if $i(\lambda([a]), \lambda([b]))=0$.

Proof Let $a$ and $b$ be two nonisotopic simple closed curves on $N$. Since $\lambda$ is injective by Lemma $3.2, \lambda([a]) \neq \lambda([b])$. Then we have the following: $i([a],[b])=0$ if and only if $[a]$ and $[b]$ are connected by an edge (since $[a] \neq[b]$ ) if and only if $\lambda([a])$ and $\lambda([b])$ are connected by an edge if and only if $i(\lambda([a]), \lambda([b]))=0$ (since $\lambda([a]) \neq \lambda([b]))$. 
In [14] the author proved that superinjective simplicial maps preserve the adjacency and nonadjacency relation with respect to top-dimensional maximal simplices in $\mathcal{C}(N)$. By following that proof and using Lemma 3.3 we get the following two lemmas:

Lemma 3.4 Suppose that $(g, n)=(3,0)$ or $g+n \geq 4$. Let $\lambda: \mathcal{C}(N) \rightarrow \mathcal{C}(N)$ be a simplicial map that satisfies the connectivity property. Let $P$ be a pair of pants decomposition on $N$ that corresponds to a top-dimensional maximal simplex in $\mathcal{C}(N)$. Let $a, b \in P$ such that $a$ is adjacent to $b$ with respect to $P$. There exists $a^{\prime} \in \lambda([a])$ and $b^{\prime} \in \lambda([b])$ such that $a^{\prime}$ is adjacent to $b^{\prime}$ with respect to $P^{\prime}$ where $P^{\prime}$ is a set of pairwise disjoint curves representing $\lambda([P])$ containing $a^{\prime}, b^{\prime}$.

Lemma 3.5 Suppose that $g+n \geq 4$. Let $\lambda: \mathcal{C}(N) \rightarrow \mathcal{C}(N)$ be a simplicial map that satisfies the connectivity property. Let $P$ be a pair of pants decomposition on $N$ that corresponds to a top-dimensional maximal simplex in $\mathcal{C}(N)$. Let $a, b \in P$ such that $a$ is not adjacent to $b$ with respect to $P$. There exists $a^{\prime} \in \lambda([a])$ and $b^{\prime} \in \lambda([b])$ such that $a^{\prime}$ is not adjacent to $b^{\prime}$ with respect to $P^{\prime}$ where $P^{\prime}$ is a set of pairwise disjoint curves representing $\lambda([P])$ containing $a^{\prime}, b^{\prime}$.

Now, by following the proofs of Lemma 2.10, Lemma 2.13, and using Lemmas 3.2, 3.3, 3.4 and 3.5, we obtain the following lemmas:

Lemma 3.6 Suppose that $g=1$ and $n \geq 3$. Let $\lambda: \mathcal{C}(N) \rightarrow \mathcal{C}(N)$ be a simplicial map that satisfies the connectivity property. If $a$ is a 1-sided simple closed curve on $N$, then $\lambda([a])$ is the isotopy class of a 1-sided simple closed curve on $N$.

Lemma 3.7 Let $g \geq 2$. Suppose that $(g, n)=(3,0)$ or $g+n \geq 4$. Let $\lambda: \mathcal{C}(N) \rightarrow$ $\mathcal{C}(N)$ be a simplicial map that satisfies the connectivity property. If $a$ is a 1 -sided simple closed curve on $N$ whose complement is nonorientable, then $\lambda(a)$ is the isotopy class of a 1-sided simple closed curve whose complement is nonorientable.

If $(g, n)=(1,2)$, there are only two vertices in the curve complex (see [30]). They are the isotopy classes of two 1 -sided curves $a$ and $b$ such that $i([a],[b])=1$, so they are not connected by an edge in $\mathcal{C}(N)$. If $\lambda$ satisfies the connectivity property then $\lambda$ could send both of these vertices to $[a]$. So, $\lambda$ does not have to be induced by a homeomorphism. In this case it is easy to see that every injective simplicial map is induced by a homeomorphism.

Theorem 3.8 Let $N$ be a compact, connected, nonorientable surface of genus $g$ with $n$ boundary components. Suppose that either $(g, n) \in\{(1,0),(1,1),(2,0)$, $(2,1),(3,0)\}$ or $g+n \geq 5$. If $\lambda: \mathcal{C}(N) \rightarrow \mathcal{C}(N)$ is a simplicial map that satisfies the connectivity property, then $\lambda$ is induced by a homeomorphism $h: N \rightarrow N$. 
Proof The proof follows as in the proof of Theorem 2.14 given by the author in [14], by using Lemmas 3.3, 3.6 and 3.7. If $(g, n)=(3,0)$ or $g+n \geq 5$, then $\lambda$ is injective by Lemma 3.2, so we also get the result by Theorem 2.15 .

\section{Acknowledgments}

We thank Mustafa Korkmaz for some discussions. We also thank the referee for some comments about the paper.

\section{References}

[1] F Atalan, Automorphisms of complexes of curves on odd genus nonorientable surfaces arXiv:math.GT/0512368

[2] F Atalan, M Korkmaz, Automorphisms of complexes of curves on nonorientable surfaces, to appear in Groups, Geometry and Dynamics

[3] J Behrstock, D Margalit, Curve complexes and finite index subgroups of mapping class groups, Geom. Dedicata 118 (2006) 71-85 MR2239449

[4] R W Bell, D Margalit, Injections of Artin groups, Comment. Math. Helv. 82 (2007) 725-751 MR2341838

[5] T E Brendle, D Margalit, Commensurations of the Johnson kernel, Geom. Topol. 8 (2004) 1361-1384 MR2119299

[6] B Farb, N V Ivanov, The Torelli geometry and its applications: Research announcement, Math. Res. Lett. 12 (2005) 293-301 MR2150885

[7] W J Harvey, Boundary structure of the modular group, from: "Riemann surfaces and related topics: Proceedings of the 1978 Stony Brook Conference", (I Kra, B Maskit, editors), Ann. of Math. Stud. 97, Princeton Univ. Press (1981) 245-251 MR624817

[8] E Irmak, Injective simplicial maps of the complexes of curves on nonorientable surfaces arXiv: 1203.4271

[9] E Irmak, Superinjective simplicial maps of complexes of curves and injective homomorphisms of subgroups of mapping class groups, Topology 43 (2004) 513-541 MR2041629

[10] E Irmak, Complexes of nonseparating curves and mapping class groups, Michigan Math. J. 54 (2006) 81-110 MR2214789

[11] E Irmak, Superinjective simplicial maps of complexes of curves and injective homomorphisms of subgroups of mapping class groups, II, Topology Appl. 153 (2006) 1309-1340 MR2202856

[12] E Irmak, Injective simplicial maps of the complex of arcs on nonorientable surfaces, Algebr. Geom. Topol. 9 (2009) 2055-2077 MR2551662 
[13] E Irmak, Simplicial maps of the complexes of curves on nonorientable surfaces (2012) arXiv:1112.1617v2

[14] E Irmak, Superinjective simplicial maps of the complexes of curves on nonorientable surfaces, Turkish J. Math. 36 (2012) 407-421 MR2993573

[15] E Irmak, N V Ivanov, J D McCarthy, Automorphisms of surface braid groups arXiv:math.GT/0306069

[16] E Irmak, M Korkmaz, Automorphisms of the Hatcher-Thurston complex, Israel J. Math. 162 (2007) 183-196 MR2365859

[17] E Irmak, J D McCarthy, Injective simplicial maps of the arc complex, Turkish J. Math. 34 (2010) 339-354 MR2681579

[18] N V Ivanov, Automorphism of complexes of curves and of Teichmüller spaces, Internat. Math. Res. Notices (1997) 651-666 MR1460387

[19] N V Ivanov, J D McCarthy, On injective homomorphisms between Teichmüller modular groups, I, Invent. Math. 135 (1999) 425-486 MR1666775

[20] Y Kida, Automorphisms of the Torelli complex and the complex of separating curves, J. Math. Soc. Japan 63 (2011) 363-417 MR2793105

[21] Y Kida, Injections of the complex of separating curves into the Torelli complex (2011) arXiv:0911.3926v3

[22] Y Kida, The co-Hopfian property of the Johnson kernel and the Torelli group, Osaka J. Math. 50 (2013) 309-337 MR3080802

[23] Y Kida, S Yamagata, Automorphisms of the Torelli complex for the one-holed genus two surface arXiv:1009.0568

[24] Y Kida, S Yamagata, Commensurators of surface braid groups, J. Math. Soc. Japan 63 (2011) 1391-1435 MR2855817

[25] Y Kida, S Yamagata, The co-Hopfian property of surface braid groups (2013) arXiv: 1006.2599v3

[26] M Korkmaz, Automorphisms of complexes of curves on punctured spheres and on punctured tori, Topology Appl. 95 (1999) 85-111 MR1696431

[27] F Luo, Automorphisms of the complex of curves, Topology 39 (2000) 283-298 MR1722024

[28] D Margalit, Automorphisms of the pants complex, Duke Math. J. 121 (2004) 457-479 MR2040283

[29] J D McCarthy, W R Vautaw, Automorphisms of Torelli groups arXiv math.GT/ 0311250

[30] M Scharlemann, The complex of curves on nonorientable surfaces, J. London Math. Soc. 25 (1982) 171-184 MR645874 
[31] K J Shackleton, Combinatorial rigidity in curve complexes and mapping class groups, Pacific J. Math. 230 (2007) 217-232 MR2318453

Department of Mathematics and Statistics, Bowling Green State University Bowling Green, $\mathrm{OH} 43403$, USA

eirmak@bgsu .edu

http://www-math.bgsu.edu/ eirmak

Received: 10 February 2013 Revised: 18 September 2013 